\title{
Arthrofibrosis Following ACL Reconstruction: Case Report
}

\author{
IGN Bagus Andhika Pramana ${ }^{1}$, IGN Wien Aryana² \\ ${ }^{1}$ Resident of Orthopaedic and Traumatology Department, Sanglah General Hospital, Udayana University, Bali \\ ${ }^{2}$ Consultant of Orthopaedics and Traumatology Department, Faculty of Medicine Udayana University, \\ Sanglah General Hospital, Indonesia \\ Corresponding Author: IGN Bagus Andhika Pramana
}

DOI: https://doi.org/10.52403/ijhsr.20220321

\begin{abstract}
Introduction: Arthrofibrosis is a debilitating complication of loss of motion after ACL reconstruction following ACL injury with a reported incidence of $2 \%$ to $35 \%$. Delayed ACL reconstruction has been the chosen management based on the suggestion from some studies of the risk reduction in developing arthrofibrosis and decreased range of motion (ROM) postoperatively.

Case Presentation: A 51-year-old female presented with complaints of pain, stiffness, and decreased range of motion following a knee ligament reconstructive arthroscopy of her right knee. After unsuccessful rehabilitative management, the patient underwent additional surgical repairs. Following the surgery, a fibrous thick scar tissue located at the suprapatellar pouch and mainly over the medial and lateral gutter was observed, in which an adhesiolysis was performed during the arthroscopic procedure. Passive range of motion of the affected knee was checked intra-operatively, showing the knee to be able to fully extend and flexed passively comparable to non affected knee. An intensive physiotherapy and continuous passive motion were begun immediately post-operatively. A 33-year-old male sustained an isolated complete tear of the left knee anterior cruciate ligament. An arthroscopy ACL single bundle reconstruction surgery with patellar tendon bone graft was done four weeks after the injury. Intraoperative was uneventful. Each end of graft was stitched together with a high strength of non-absorbable suture. Graft fixations used bio absorbable screws and loaded with extracortical buttons. After post operation, patient was observed to be able to perform full active knee extension. The patient was very compliant to rehabilitation program and also performed the exercises at home. However, on clinic follow-up, the patient started to gradually loss his active full extension and on subsequent visit further affected his passive full extension (10 degrees extension lag) at 4 months post operation. Regional examination showed diffused firm swelling over the anteromedial aspect of the knee just above the medial joint line that was very tender. An intensive physiotherapy and continuous passive motion were begun immediately post operatively.

Result: In the first patient, the functional outcome score showed improvement at the time of final followup, as well as the average pain scores. This case report emphasized on the importance of early recognition as well as assessment by the orthopaedic practitioners of arthrofibrosis as a complication of ACL reconstructive surgery is the key point in reducing the long-term morbidity caused by the condition in patients unable to regain flexibility at the expected rate after injury or surgical treatment. Rehabilitation protocols should mainly stress on early motion, rapid return of quadriceps function and excursion, and patella mobilization. The second patient, functional outcome was measured with KSS to assess pain and function after arthroscopy. The functional outcome score showed improvement from 68 points preoperatively to 86 at the time of final follow-up. The average pain scores improved as well from 30 points pre-operatively to 41 at the time of final follow-up.

Conclusion: Arthrofibrosis following ACL reconstruction represents a broad continuum of abnormalities, and hence a thorough understanding of the condition and a comprehensive approach to its management is of importance to achieve a functional result.
\end{abstract}

Key Word: Arthrofibrosis, Loss of motion, ACL Reconstruction, Arthroscopy 


\section{INTRODUCTION}

Arthrofibrosis is a debilitating complication of loss of motion after ACL reconstruction following ACL injury with a reported incidence of $2 \%$ to $35 \% .{ }^{1}$ It is a multifactorial condition, involving a combination of mechanical and biologic factors, which some of them being surgical technique, timing of surgery, and prolonged immobilization. While early recognition is likely to be the most important intervention, treatment ranges from prevention and frequent supervised physical therapy to surgical procedure. Delayed ACL reconstruction has been the chosen management instead of the acute one due to the suggestion from some studies of the risk reduction of developing arthrofibrosis and decreased range of motion (ROM) postoperatively. However, other studies from Wasilewski et al and Bottoni et al in a randomized controlled trial of patients undergoing ACLR with hamstring graft beg to differ. The studies resulted in no significant difference in extension or flexion loss comparing surgery within 21 days of injury to surgery after 6 weeks. In most cases the patient with postoperative limited range of motion has poorer function on activity daily living. While the treatment of arthrofibrosis can be quite bothersome to the patient as well as to the orthopaedic surgeon, a thorough understanding of the condition and a comprehensive approach to its management is of importance to achieve a functional result.

\section{CASE REPORT}

\section{Patient-1}

\section{History and Examination}

A 51 year-old female came to polyclinic of Sanglah General Hospital with complaints of pain and stiffness on the right knee after undergoing knee ligament reconstruction surgery since three months before admission. Patient had a history of falling in the bathroom with the right leg withstanding her body, in which she was undergoing ACL+MCL reconstruction surgery for being diagnosed with tears of
MCL and ACL of his right knee as well as tears of meniscus. Reconstruction surgery was done three weeks after the injury. ACL and MCL reconstruction were performed arthroscopically with anatomical single bundle reconstruction with hamstring graft. The hamstring graft was taken from the ipsilateral semitendinosus and gracilis tendon prepared into two-strand graft with length of $28 \mathrm{~cm}$ and diameter of $0.8 \mathrm{~cm}$. Each end of graft was stitched together with a high strength of non-absorbable suture and fixated with $9 \times 25 \mathrm{~mm}$ bio absorbable screws. Post-operative follow-up showed that patient was able to perform full active knee extension. Rehabilitation started immediately post-operative where the patient's knee was put on full extension with the help of a pillow under the ankle.

On post-operation day one, active ROM exercises were taught to patients, including ankle pump exercise, passive quadriceps strengthening, straight leg raised, patella mobility and knee active-assisted ROM exercise. The knee was kept in full extension except during ROM exercises. The patient was allowed to weight-bear because there was no concomitant meniscal repair procedure done. She was very compliant to rehabilitation program and also performed the exercises at home.

Post-operative follow-ups were scheduled starting from week 2 . The range of motion was examined every follow-ups. The patient was observed to start to gradually loss her active and passive full extension beginning in 3 months follow up from $5^{0}$ to $10^{0}$ extension lag, in which she was then planned to undergo aggressive rehabilitation. However, in 2 weeks of rehabilitation, the patient's current condition didn't progress well. Thus, the patient underwent manipulation under anaesthesia (MUA), before debridement arthroscopy was performed. The patient's knee was initially unable to be fully extended, but then the passive range of motion progressed from $10^{\circ}$ to $120^{\circ}$. Her flexion was almost equal compared to opposite knee. And there was a lack of extension in her right knee. 
In durante-op, a fibrous thick scar tissue located at the suprapatellar pouch and mainly over the medial and lateral gutter was observed, with a meniscus tear as well. There was no loose body or cyclope lesion found. Adhesiolysis was performed during the arthroscopic procedure. Passive range of knee motion was checked intraoperatively, which showed the knee to be able to be fully-extended and flexed passively comparable to non affected knee.
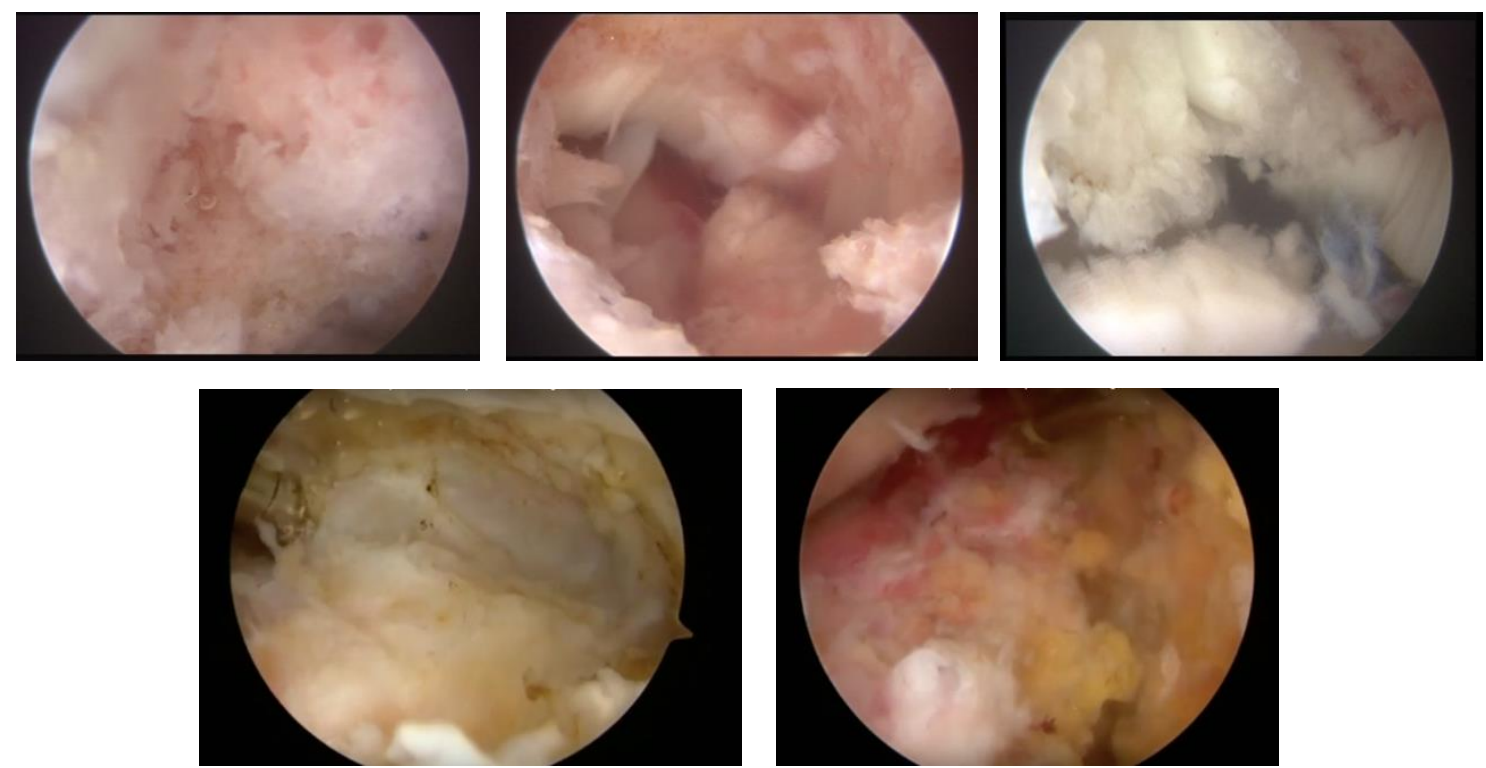

Figure 1. Debridement of adhesions has performed on the right knee

\section{Patient-2}

\section{History and Examination}

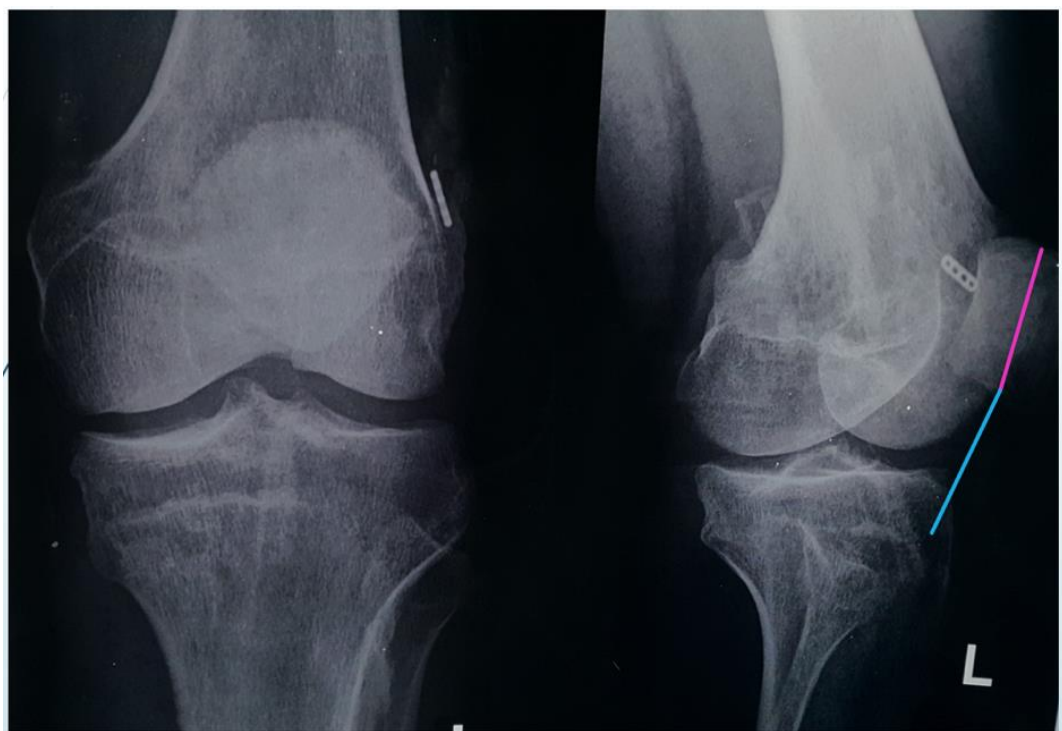

Figure 2. Left knee X-ray with Insall-Salvati Ratio 1,2 following ACL Reconstruction

A 33-year-old male sustained an isolated complete tear of the left knee anterior cruciate ligament. An arthroscopy ACL single bundle reconstruction surgery with patellar tendon bone graft was done four weeks after the injury. Intraoperative was uneventful. Each end of graft was stitched together with a high strength of non-absorbable suture. Graft fixations used bio absorbable screws and loaded with extracortical buttons. After post operation, patient was observed to be able to perform full active knee extension. The patient was very compliant to rehabilitation program 
and also performed the exercises at home. However, on clinic follow-up, the patient started to gradually loss his active full extension and on subsequent visit further affected his passive full extension (10 degrees extension lag) at 4 months post operation. Regional examination showed diffused firm swelling over the anteromedial aspect of the knee just above the medial joint line that was very tender.

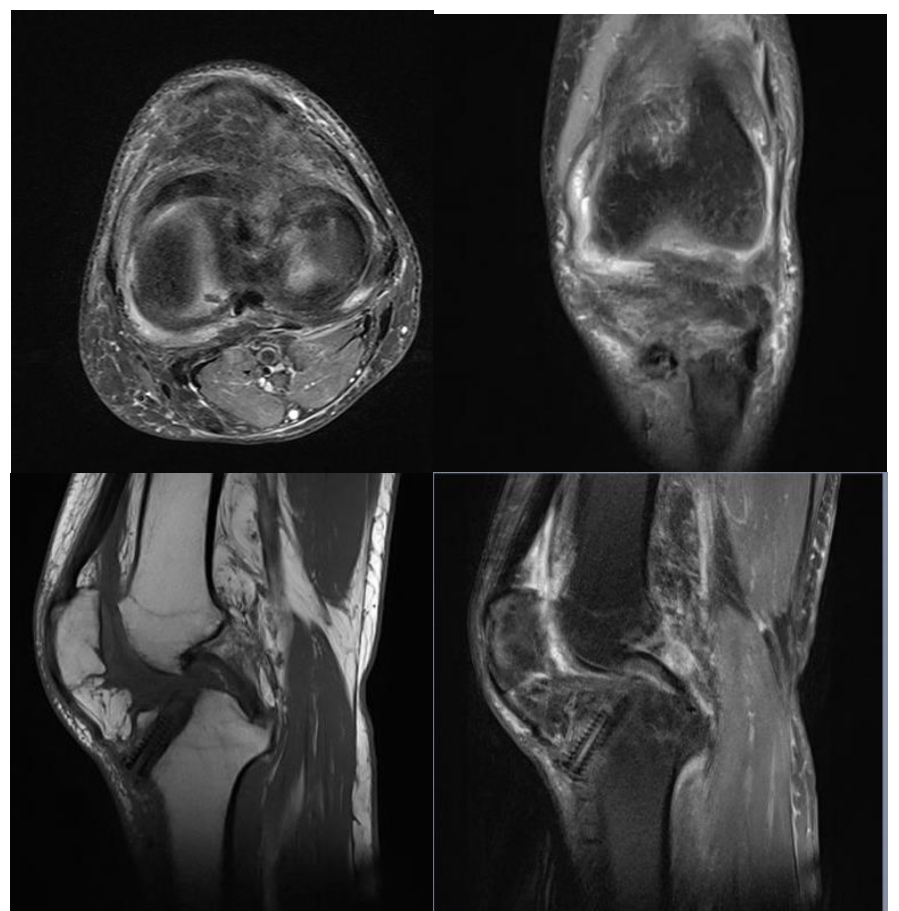

Figure 2. Left knee MRI from axial. Sagittal, and coronal view showed there is an Arthrofibrosis following ACL Reconstruction

Before diagnostic arthroscopy procedure was done, manipulation under anaesthesia (MUA) was performed. His knee was initially unable to be fully extended. The range of motion passively was from $10^{\circ}$ to $140^{\circ}$. His flexion was almost equal compared to opposite knee. From arthroscopy diagnostic we found thick scar tissue located at the suprapatellar pouch. Debridement of adhesions was performed during the arthroscopic procedure which then range of knee motion was checked intraoperatively, showing the knee to be able to be fully-extended and flexed passively comparable to non-affected knee.

An intensive physiotherapy and continuous passive motion were begun immediately post operatively. Functional outcome was measured with KSS to assess pain and function after arthroscopy. The functional outcome score showed improvement from 68 points pre-operatively to 86 at the time of final follow-up. The average pain scores improved as well from 30 points pre-operatively to 41 at the time of final follow-up.

\section{DISCUSSION}

Arthrofibrosis was first described by Luck as the loss of range of motion secondary to intraarticular scar tissue formation. While Hughston in 1985 described three types of this condition, including fibrosis in the intercondylar notch; secondary fibrosis of the supra-patellar pouch; and fibrosis in the lateral and supracondylar area, which subsequently was described by many orthopaedists as limited range of motion from intraarticular and extraarticular fibrosis after open and endoscopic procedures. ${ }^{7}$ The etiology of arthrofibrosis is multifactorial. Potential causes involving a combination of mechanical and biologic factors, include: surgical technique, timing of surgery, prolonged immobilization, rehabilitation, additional ligamentous surgery, meniscal 
repair procedures, infection, reflex sympathetic dystrophy, and complex regional pain syndrome. ${ }^{7,13}$

Arthrofibrosis can occur after surgical as well as nonsurgical injury to the knee, which is usually associated with both extension and flexion loss. While graft placement anterior to the native ACL insertion on the tibia results in impingement on the roof of the intercondylar notch in extension, lateral placement on the tibia produces impingement on the lateral wall of the intercondylar notch. Furthermore, placement too far anteromedially has been shown to limit flexion. Most importantly, on the femoral side, the most common error is graft placement too far anterior, which causes excessive strain on the graft, leading to limited flexion and potential graft failure. ${ }^{7,13}$ Meanwhile, the role of timing of surgery as one of the risk factors of arthrofibrosis remain controversial. The landmark study by Shelbourne et al is the primary source for the recommendation to differentiate acute from delay ACL reconstruction (ACLR) following an acute rupture by at least 3 weeks delay from initial injury. ${ }^{10}$ The study reviewed 169 young athletes after acute ACLR, which had the results of statistically significant increased incidence of arthrofibrosis in patients whose ACL ligament reconstruction was done within 1 week of injury $(\mathrm{P}<0.05)$ compared to those of patients' ACL reconstruction had been delayed by $>3$ weeks. Other study by Harner et al also found a $37 \%$ rate of postoperative motion loss after acute ACLR (16 of 43 patients) versus 5\% after chronic reconstruction. ${ }^{14}$ While Wasilewski et al evaluated 87 patients who underwent reconstruction for ACL injury at the acute ( $<1$ month), subacute (1 to 6 months), and chronic ( $>6$ months) stage. However, these studies were retrospective, and other studies have indicated surgical timing as less of an effect to the occurrence of arthrofibrosis. ${ }^{15}$

Mayr et al retrospectively reviewed a cohort of 156 patients with post-operative arthrofibrosis and found significant correlations between knee irritation, effusion and swelling and development of arthrofibrosis, rather than the timing of surgery. ${ }^{16}$ The rationale for acute reconstruction is that if the surgery is performed within the first few days after the injury, the surgical trauma itself will blend into the trauma from the injury. In contrast, a slightly delayed operation when healing is already underway may result in a second hit, due to surgical trauma, resulting in an increased risk for arthrofibrosis. A level IIof-evidence study of Erikkson et al stated that acute reconstruction did not result in increased stiffness. ${ }^{17}$ The acute group had better one leg hop tests, better improvements within the subscales pain, symptoms and quality of life in KOOS, and better outcomes regarding how the knee affected their activity level at 6 months. Additionally, the acute group was not inferior to the delayed group in any assessment. These findings are supported by the systematic review by Andernord et al. ${ }^{18}$ Immobilization may seem to allow ligamentous healing; however, the functional result and load to failure for the healed ligament is poor. $^{7}$ In 1991, Shelbourne et a1 reported the incidence of arthrofibrosis was decreased by early active and passive knee extension, progressive physical therapy and appropriate surgical timing. Shelbourne et al and other orthopaedists subsequently showed that early controlled motion and weight bearing beginning on postoperative day one, as well as supervised physical therapy with progression of weight bearing, resulted in better range of motion in the early postoperative period. ${ }^{10}$

As for the classification of arthrofibrosis, various orthopaedists have extensively described and classified arthrofibrosis and recommended treatment parameters. Shelbourne et a1 interpreted arthrofibrosis as any loss of knee motion compared with the normal contralateral knee that is symptomatic, which then subdivided arthrofibrosis into four types based on severity of motion loss to assist in determining the appropriate treatment 
(Table 1). ${ }^{11}$ A severe form of arthrofibrosis that results in shortening of the patellar tendon and patella baja has been described by Paulos et al who termed this type of arthrofibrosis as infrapatellar contracture syndrome (IPCS), divided into three stages: (1) the prodromal stage from 2 to 8 weeks, which responded best to active intervention;

(2) the active stage from 6 to 20 weeks; and (3) the burned-out stage. ${ }^{19}$

\begin{tabular}{|c|c|c|}
\hline Type & $\begin{array}{l}\text { Extension } \\
\text { Loss }\end{array}$ & Flexion Loss \\
\hline 1 & $<10^{\circ}$ loss & Normal flexion \\
\hline 2 & $>10^{\circ}$ loss & Normal flexion \\
\hline 3 & $>10^{\circ}$ loss & $>25^{\circ}$ loss with tight patella \\
\hline 4 & $>10^{\circ}$ loss & $\begin{array}{l}>30^{\circ}, \text { patella infera with marked } \\
\text { patellar tightness }\end{array}$ \\
\hline
\end{tabular}

The term arthrofibrosis describes a wide spectrum of conditions, which ultimately result in motion loss. Fullerton and Andrews ${ }^{11}$ first reported hypertrophy of the ACL graft and protrusion of bone at the tibial attachment site. Jackson and Schaefer ${ }^{12}$ further characterized this finding, now known as "cyclops syndrome," as extension loss after ACL reconstruction caused by a mechanical block from hypertrophic fibrous tissue attached to the tibial insertion point of the ACL graft (Figure 1), which is the impingement on the top of the notch and graft hypertrophy with fibrous tissue proliferation resulting from a repetitive trauma of graft against bone caused by anterior graft placement.

Infrapatellar contraction syndrome (IPCS) is another clinical finding of arthrofibrosis, manifesting as significant reduction of both flexion $\left(>25^{\circ}\right)$ and extension $\left(>10^{\circ}\right)$ with an associated decrease in patellar mobility, characterized as patellar entrapment. ${ }^{2}$ Paulos et $\mathrm{al}^{19}$ stratified patients with IPCS into prodromal, active, and residual stages. A patient in the prodromal stage demonstrates periarticular inflammation and edema, quadriceps weakness and lag, poor knee extension, painful ROM, and tenderness over the patellar tendon with a decrease in patellar excursion. A patient in the active stage shows a dramatic decrease in patellar mobility, marked quadriceps atrophy, worsening knee motion, and fat pad induration with a rigid patellar tendon. A patient in the residual stage presents with peripatellar and retinacular tissues that are more supple than in the active stage, with resulting marked quadriceps atrophy and loss of knee flexion and extension. The critical component of the residual stage is the progression of patellofemoral arthrosis and patella infera (Figure 2). ${ }^{13}$

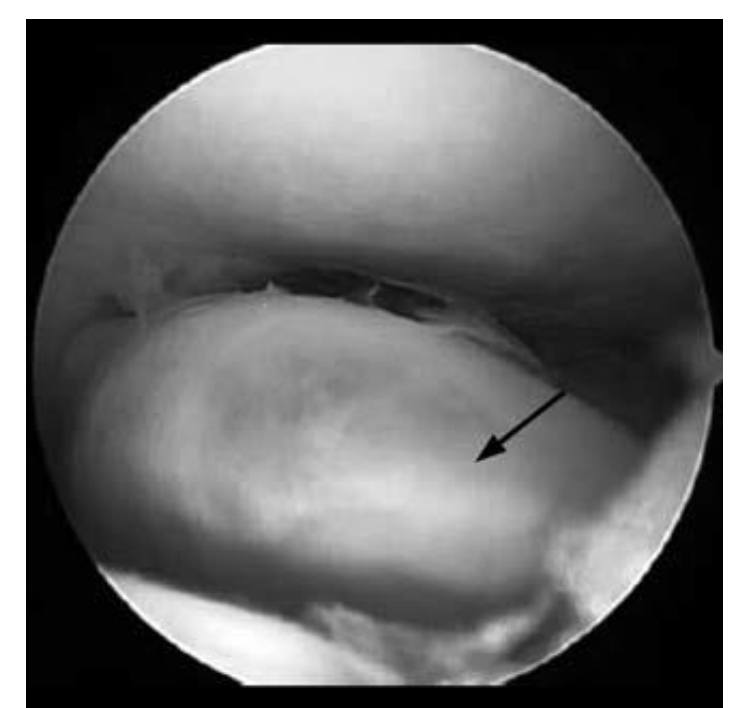

Figure 1. Cyclops lesion (arrow) located anterior to the intercondylar notch. (Reproduced with permission from Ahn JH, Yoo JC, Yang KS, Kim JH, Wang JH:mSecond-look arthroscopic findings of 208 patients after ACL reconstruction. Knee Surg Sports Traumatol Arthrosc 2007;15:242-248.)

Prevention, as important as that of other diseases, is the most effective choice of avoiding the debilitating consequences of motion loss after knee ligament injury and surgery. Improved postoperative rehabilitation protocols, including the use of immediate passive and progressive active ROM, have addressed the harmful consequences of prolonged immobilization (Figure 3). After determining the patient's clinically significant arthrofibrosis, using the criteria established by Shelbourne9, the type of arthrofibrosis present need to be classified. 


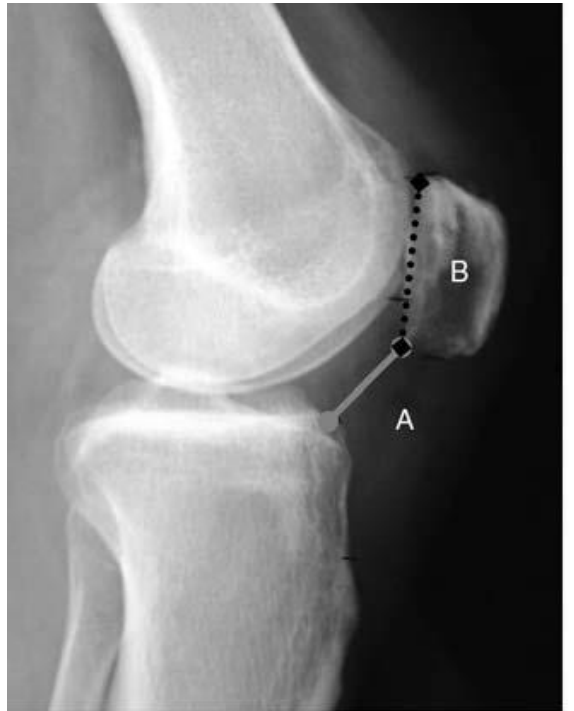

A

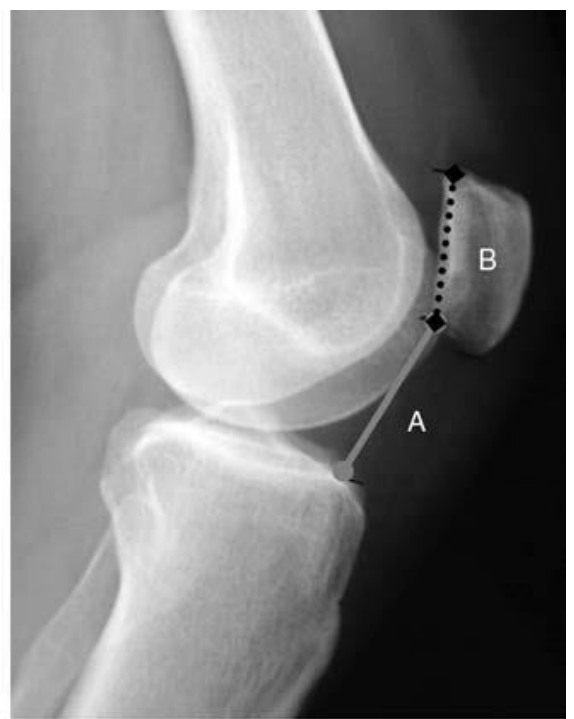

B

Figure 2. Lateral radiographs demonstrating patella infera after patellar tendon repair. The Blackburne-Peel index is shown on presentation $(A / B=0.62)(A)$ and after gait retraining and quadriceps reactivation $(A / B=1.18)(B)$.

There are several references with parameters of motion to achieve and their time period to achieve motion goals (Table 2). ${ }^{1}$ Shelbourne ${ }^{9}$ has reported recently on surgical treatment of arthrofibrosis by his classification. Type 1 patients underwent simple cyclops excision at the base of the ACL graft until it fit in the notch without any impingement with the knee in full extension. Type 2 patients involved resection of the anterior scar, extra-synovial scar anterior to the proximal tibia, and a thick layer of tissue over the ACL graft, which additionally, further notchplasty was performed to ensure no residual impingement existed. An adequate space between the ACL graft and the notch was as well achieved intraoperatively. Type 3 patients underwent resection of the scar between the fat pad and the patellar tendon and proximally both medially and laterally to the tendon insertions to free the patella and patellar ligament, A blunt probe was used to establish the plane of the patellar tendon and the scar tissue to be resected. The arthroscopic scar resection included removal of scar tissue distal to the upper tibia and anterior to the horns of both menisci. Once the anterior tibial and retropatellar tendon scar tissue were resected, the fibrotic capsule was excised up to the vastus medialis obliquus and the vastus lateralis tendon insertions to free the patella and the patellar tendon. While graft placement was then analyzed to be too far anteriorly, the anterior fibers of the graft were resected and notchplasty was performed laterally and anteriorly to enable the graft to fit into the intercondylar notch to achieve full extension. Additional medial and lateral scar resection was required for Type 4 patients to completely release the patella and patellar tendon. It is necessary for patients with Type 3 and 4 arthrofibrosis to be performed a knee manipulation after the scar resection to achieve full flexion (Table 3). ${ }^{1,9}$

Several author (K.E.D.) advocates two additional preventive measures after surgical treatment of symptomatic arthrofibrosis. First, the intraoperative and postoperative use of steroids transitioning to NSAIDs to help prevent recurrent arthrofibrosis.1 Secondly, the patients were to placed in a full-cylinder cast in full extension postoperatively, which is bivalved to allow intermittent ( 4 to 5 times a day) flexion range of motion (ROM) starting the first postoperative day. This is continued for the first 2 weeks, keeping the cast on between ROM sessions, where the cast is worn only at night to prevent development of recurrent flexion contracture overnight. ${ }^{1}$ 
In the immediate postoperative motion, intraarticular drains are essential to reduce the potential complication for haemarthrosis. Early motion; including CPM, cryotherapy, and regional pain management techniques, such as femoral nerve block or an indwelling epidural catheter, should all be done. Daily physical therapy for patella mobilization, joint motion, and recruitment of the quadriceps is indicated, often for the first few weeks. There is often a biphasic improvement in joint motion, with immediate postoperative motion decreasing over the next 7 to 10 days in the early inflammatory phase of healing. As the warmth and inflammation fade, motion can again regained. Oral corticosteroids, in a high-dose tapered course over 3 to 4 weeks, can be of significant benefit for patients with significant post release inflammation. Because of the significant cortical defect created just distal to the tibial tubercle by the DeLee osteotomy, even when grafted, these patients require long-term protection with crutches and a hinged brace, often up to 12 weeks postoperatively. This area is also prone to stress reactions or stress fractures if the patient returns to running, even after complete healing. ${ }^{1-3}$

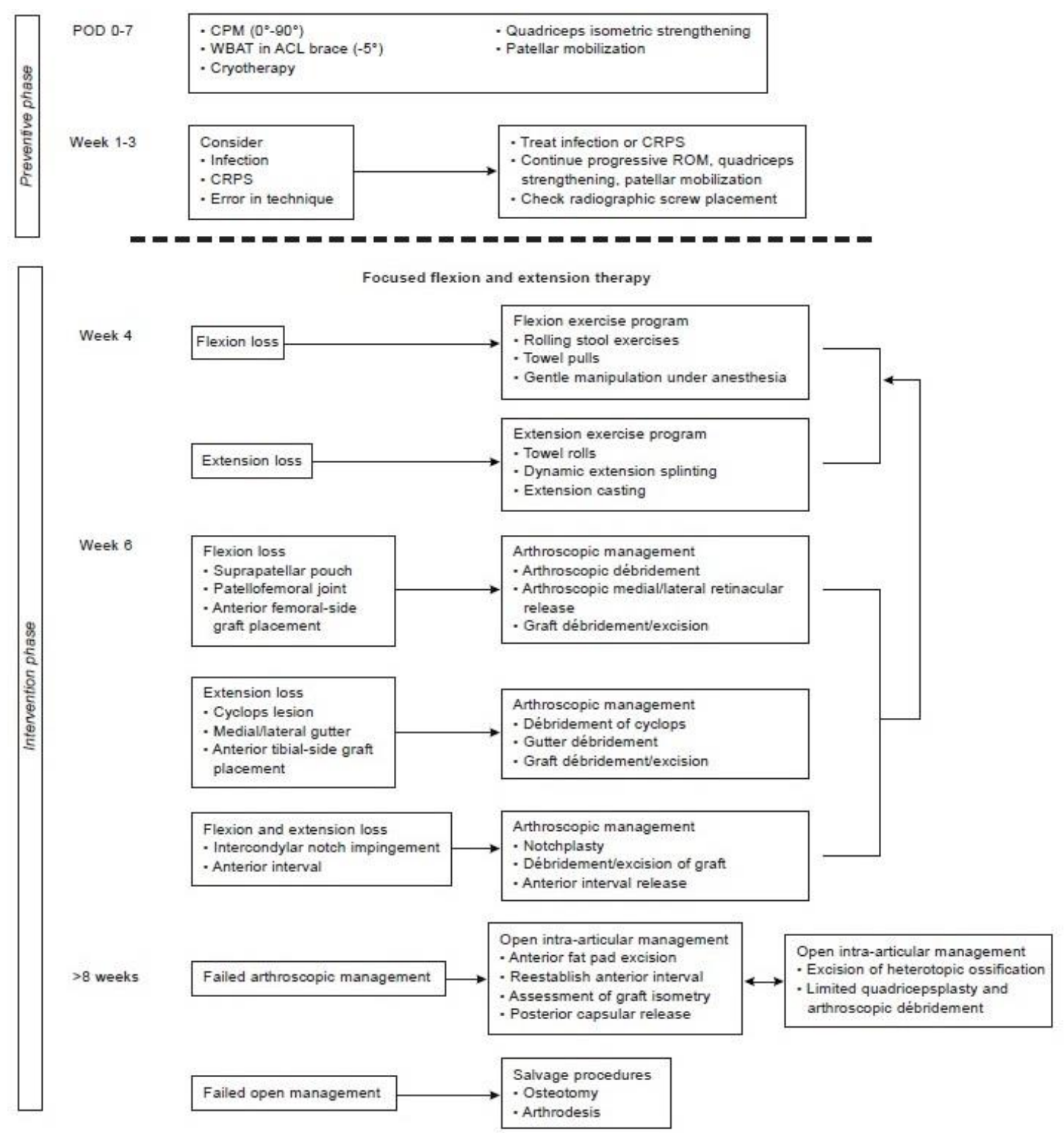

Figure 3. Treatment algorithm for arthrofibrosis highlighting prevention (top) and intervention (bottom) postoperatively. ACL $=$ anterior cruciate ligament, CPM = continuous passive motion, CRPS = complex regional pain syndrome, $P O D=$ postoperative day, ROM = range of motion, WBAT = weight bearing as tolerated. 
Postoperative outcomes are quite variable to interpret, given the different classification systems, variable injury patterns, combined surgical treatment patterns, and different outcome measures. There are several reports on the results of severely arthrofibrotic knees that have undergone multiple surgical treatments. Millett et $\mathrm{al}^{14}$ reported on eight patients with an average preoperative ROM of $62.5^{\circ}$ who underwent open debridement. After radical open debridement (mean follow-up, 57 months), patients gained an average of $62^{\circ}$ of motion. However, in five of eight patients, degenerative changes were seen on radiographs at a mean follow-up of 57 months. All patients were able to return to sports, but only one was able to achieve her preinjury level of function. ${ }^{2,14}$

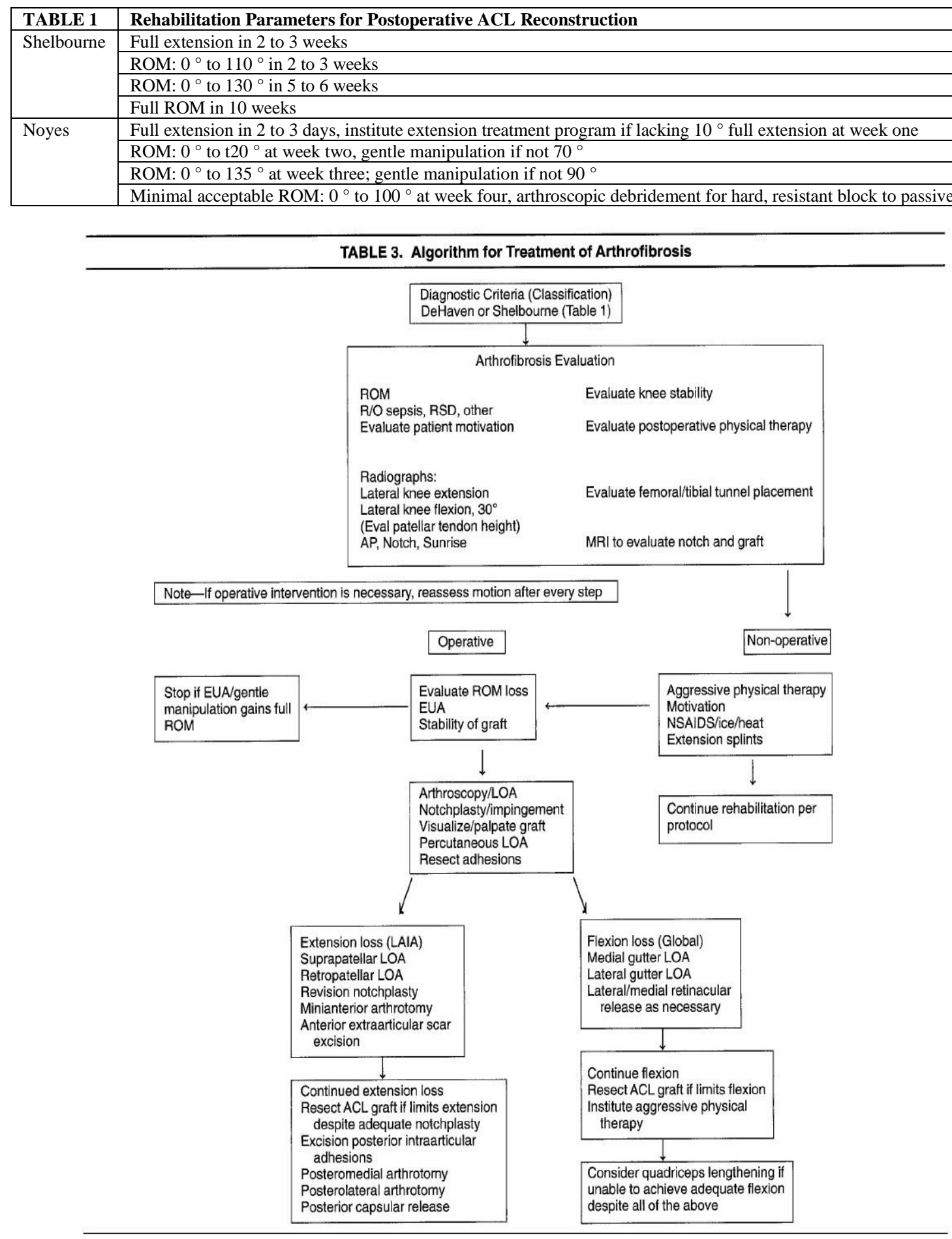


Early assessment, and thus, recognition by the orthopaedic practitioners of patients whom unable to regain flexibility at the expected rate after injury or surgical treatment is the key point in reducing the long-term morbidity caused by the condition as well as its complication. The precursor of motion issues, which one of the many being pain that is greater than anticipated pain, should be an alert for the practitioners of this particular issue.

Abbreviations: R/O, rule out; RSD, reflex sympathetic dystrophy; EUA, exam under anesthesia; PT, physical therapy; LOA, lysis of adhesions.

\section{CONCLUSION}

Arthrofibrosis remains a potentially debilitating complication of ACL reconstruction following an acute ACL injury. It is a condition of multifactorial etiology and its occurrence needs rapid identification to initiate appropriate modalities of prevention and treatment, which the initial step being the classification of the condition into 4 types of arthrofibrosis established by Shelbourne et al, by which will guide one to the appropriate treatment plan (nonoperative and operative). The timing of surgery of ACL reconstruction within the acute phase (less than 3 weeks) has been shown by some studies to have a hinger incidence of arthrofibrosis, the studies advocated that ACL reconstruction should be delayed until adequate ROM is achieved (preinjury or within $5^{\circ}$ of full extension and more than $90^{\circ}$ flexion), and the posttraumatic inflammatory phase has resolved. Whereas other studies stated that acute ACL reconstruction can be performed safely without an increased risk of developing decreased range of motion, by whom the patients undergoing acute reconstruction within 8 days did not result in reduced ROM compared to delayed surgery, but on the contrary had significantly less muscle hypotrophy in the early phase of the rehabilitation and significantly better one leg hop test. After surgical treatment of arthrofibrosis, rehabilitation protocols should stress early motion (particularly full passive extension, if it was limited preoperatively), rapid return of quadriceps function and excursion, and patella mobilization.

Arthrofibrosis following ACL reconstruction represents a broad continuum of abnormalities, and hence a thorough understanding of the condition and a comprehensive approach to its management is of importance to achieve a functional result.

\section{Acknowledgement: None}

\section{Conflict of Interest: None}

\section{Source of Funding: None}

\section{REFERENCES}

1. Gianotti SM, Marshall SW, Hume PA, Bunt L. Incidence of anterior cruciate ligament injury and other knee ligament injuries: A national population-based study. J Sci Med Sport. 2009. doi:10.1016/j.jsams.2008.07.005

2. Janssen KW, Orchard JW, Driscoll TR, van Mechelen W. High incidence and costs for anterior cruciate ligament reconstructions performed in Australia from 2003-2004 to 2007-2008: Time for an anterior cruciate ligament register by Scandinavian model? Scand J Med Sci Sport. 2012. doi:10.1111/j.1600-0838.2010.01253.x

3. Buller LT, Best MJ, Baraga MG, Kaplan LD. Trends in anterior cruciate ligament reconstruction in the United States. Orthop J Sport 2015. doi:10.1177/2325967114563664

4. St. Pierre P. Complications of anterior cruciate ligament surgery. Sports Med Arthrosc. 2004. doi:10.1097/01.jsa.0000131852.82203.b4

5. Vergis A, Gillquist J. Graft failure in intraarticular anterior cruciate ligament reconstructions: A review of the literature. Arthrosc J Arthrosc Relat Surg. 1995. doi:10.1016/0749-8063(95)90009-8

6. Jaureguito JW, Greenwald AE, Wilcox JF, Paulos LE, Rosenberg TD. The incidence of deep venous thrombosis after arthroscopic 
knee surgery. Am J Sports Med. 1999. doi:10.1177/03635465990270060401

7. Gillespie MJ, Friedland J, Dehaven KE. Arthrofibrosis: Etiology, classification, histopathology, and treatment. Oper Tech Sports Med. 1998. doi:10.1016/S10601872(98)80057-9

8. Sprague NF, O'Connor RL, Fox JM. Arthroscopic treatment of postoperative knee fibroarthrosis. Clin Orthop Relat Res. 1982. doi:10.1097/00003086-19820600000029

9. Ekhtiari S, Horner NS, de Sa D, et al. Arthrofibrosis after ACL reconstruction is best treated in a step-wise approach with early recognition and intervention: a systematic review. Knee Surgery, Sport Traumatol Arthrosc. 2017. doi:10.1007/s00167-017-4482-1

10. Shelbourne KD, Wilckens JH, Mollabashy A, Decarlo M. Arthrofibrosis in acute anterior cruciate ligament reconstruction: The effect of timing of reconstruction and rehabilitation. Am J Sports Med. 1991. doi:10.1177/036354659101900402

11. Shelbourne KD, Patel D V., Martini DJ. Classification and management of arthrofibrosis of the knee after anterior cruciate ligament reconstruction. Am $J$ Sports Med. 1996. doi:10.1177/036354659602400625

12. Noyes FR, Berrios-Torres S, Barber-Westin SD, Heckmann TP. Prevention of permanent arthrofibrosis after anterior cruciate ligament reconstruction alone or combined with associated procedures: A prospective study in 443 knees. Knee Surgery, Sport Traumatol Arthrosc. 2000. doi:10.1007/s001670000126

13. Haklar U, Ayhan E, Ulku TK, Karaoglu S. Arthrofibrosis of the Knee. In: Sports Injuries: Prevention, Diagnosis, Treatment and Rehabilitation, Second Edition. ; 2015. doi:10.1007/10.1007/978-3-642-365690_74

14. Harner CD, Irrgang JJ, Paul J, Dearwater S, $\mathrm{Fu} \mathrm{FH.} \mathrm{Loss} \mathrm{of} \mathrm{motion} \mathrm{after} \mathrm{anterior}$ cruciate ligament reconstruction. Am $J$ Sports Med. 1992. doi:10.1177/036354659202000503

15. Wasilewski SA, Covall DJ, Cohen S. Effect of surgical timing on recovery and associated injuries after anterior cruciate ligament reconstruction. Am J Sports Med. 1993. doi:10.1177/036354659302100302

16. Mayr HO, Weig TG, Plitz W. Arthrofibrosis following ACL reconstruction - Reasons and outcome. Arch Orthop Trauma Surg. 2004. doi:10.1007/s00402-004-0718-x

17. Eriksson K, von Essen C, Jönhagen S, Barenius B. No risk of arthrofibrosis after acute anterior cruciate ligament reconstruction. Knee Surgery, Sport Traumatol Arthrosc. 2018. doi:10.1007/s00167-017-4814-1

18. Andernord D, Karlsson J, Musahl V, Bhandari M, Fu FH, Samuelsson K. Timing of surgery of the anterior cruciate ligament. Arthrosc - J Arthrosc Relat Surg. 2013. doi:10.1016/j.arthro.2013.07.270

19. Paulos LE, Wnorowski DC, Greenwald AE. Infrapatellar Contracture Syndrome: Diagnosis, Treatment, and Long-Term Followup. Am J Sports Med. 1994. doi:10.1177/036354659402200402

How to cite this article: IGN Bagus Andhika Pramana, IGN Wien Aryana. Arthrofibrosis following ACL reconstruction: case report. Int $J$ Health Sci Res. 2022; 12(3): 149-159. DOI: https://doi.org/10.52403/ijhsr.20220321 